\title{
PAGES2k: Advances in climate field reconstructions
}

\author{
Kevin J Anchukaitis ${ }^{1}$ and Nicholas McKay ${ }^{2}$ \\ Woods Hole, United States, 15-16 April 2014
}

A major outcome of the PAGES2k synthesis was the creation of continental-scale reconstructions of mean regional temperatures that span the last millennium or more (PAGES $2 k$ Consortium 2013). Four of the PAGES $2 \mathrm{k}$ regions (Asia, Europe, North and South America) also reconstructed spatial variability in temperatures using approaches that fall within a class of methodologies called climate field reconstruction (CFR; Evans 2001). CFR consists of a set of statistical tools that can be used to estimate past climate variability on a regular grid, typically using networks of annual-resolution paleoclimate proxies. They exist on a continuum from simple reconstructions of a single leading spatiotemporal mode (analogous to a weighted mean reconstruction) to more complex methods including hierarchical models that incorporate knowledge of the proxy systems and climate covariance (Tingley and Huybers 2010; Steiger et al. 2014). These reconstructions are desirable as they allow climate variability to be estimated in both space and time, providing targets for general circulation model comparisons and knowledge about the fingerprint of regional-scale climate variability in response to radiative forcing and internal climate system variability.

The PAGES2k proxy dataset provides specific challenges for CFR. The data are highly heterogeneous in space and time, they are known to contain a range of frequency-dependent, seasonal, multivariate climate signals, and include proxy archives that are lower resolution and time-uncertain. During the Phase I synthesis of the PAGES2k project, we identified three primary methodological challenges to developing field reconstructions across all the regional networks. These included (1) the need to develop unbiased approaches to the selection of proxy data from a large database of potential predictors, (2) the incorporation of non-annual and time-uncertain records in climate reconstructions (e.g. Anchukaitis and Tierney 2012), and (3) the complications presented by the PAGES2k Phase II goal to reconstruct hydroclimate, specifically with respect to seasonality and spatial covariance.

The PAGES2k Advances in Climate Field Reconstruction workshop brought together paleoclimatologists from across the $2 k$ Network with experts on reconstruction methods to explore these questions and to work toward developing new techniques that address extant challenges. The workshop included a thorough overview from proxy domain experts on the spatial, temporal, and spectral characteristics of different proxy systems, including sources of uncertainty and bias. Existing CFR methods were reviewed in light of their ability to be adapted specifically to the PAGES2k network. A major feature of the workshop was the initiation of a reconstruction methods intercomparison exercise. Using the Arctic 2k data (McKay and Kaufman 2014), researchers representing different CFR approaches were given the actual proxy data as well as a set of pseudoproxies (Smerdon 2012) designed to mimic the spatial, temporal, seasonal, and chronological characteristics of the real network. The goal was to observe differences
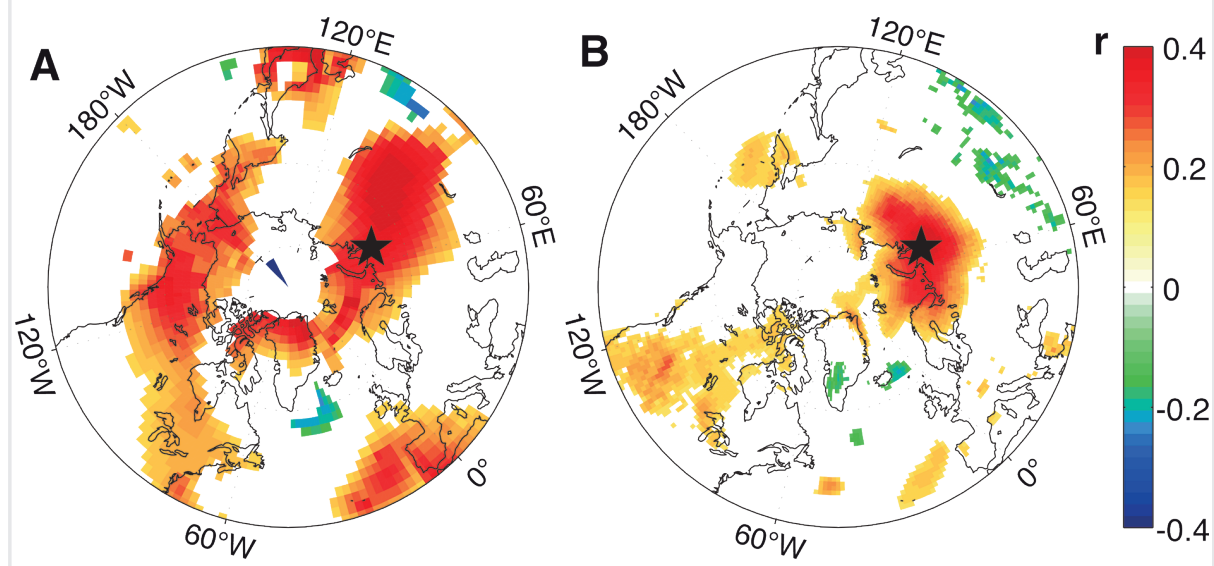

Figure 1: Correlation between March-August mean temperatures and (A) simulated pseudoproxy and (B) actual tree-ring chronologies from the Yamal Peninsula (Briffa et al. 2008; McKay and Kaufman 2014). The pseudoproxy series was created using simulated temperatures from the last millennium NCAR CCSM4 simulation with additional Gaussian noise to mimic the signal-to-noise ratio of the actual chronology. Correlations for the pseudoproxy series are with the corresponding CCSM4 mean surface temperature field, while the actual Yamal chronology is correlated against the GISTEMP (Hansen et al. 2010) combined land-sea temperature field. The star indicates the location of the chronology.

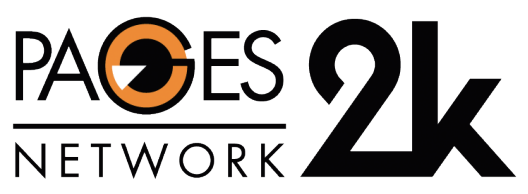

in estimates of past climate variability as a function of reconstruction method and to provide a testbed for evaluating their origin. Initial efforts exposed practical challenges posed by the sparseness of the network, the signal-to-noise signature of the proxies, and time-uncertainty. These also provided information about the design of more realistic pseudoproxy experiments (e.g. Fig 1). The goal of creating a global reconstruction using the PAGES2k database was also explored.

The workshop developed several concrete goals and recommendations, including: continued development of database structures that allow for expert and community assessment to facilitate open discussion of proxy strengths, weaknesses, biases, selection, and fidelity, a prerequisite for future regional and global reconstructions; continued and expanded development of proxy system models (Evans et al. 2013) for capturing the multivariate, nonlinear, and seasonal aspects of different proxies; incorporation of explicit hierarchical modeling of chronological uncertainty within CFR techniques; and exploration of CFR approaches that transparently integrate scientific knowledge of the proxies and climate systems within the statistical modeling framework (Tingley et al. 2012). Based on initial efforts toward a methods intercomparison, the group has designed a new series of experiments to isolate the influence of model specification, climate signal, network distribution, and time uncertainty. Methods development and testing with respect to incorporating time uncertainty within hierarchical models is ongoing, and a community effort to develop an improved database and a global-scale reconstruction is proceeding.

\section{AFFILIATIONS}

'Department of Geology and Geophysics, Woods Hole Oceanographic Institution, Woods Hole, USA 'School of Earth Sciences and Environmental Sustainability, Northern Arizona University, Flagstaff, USA

\section{CONTACT}

Kevin Anchukaitis: kja@whoi.edu

\section{REFERENCES}

Evans MN et al. (2001) In: Markgraf V (Ed)

Interhemispheric Climate Linkages, Cambridge, 53-72

Evans MN et al. (2013) Quat Sci Rev 76: 16-28

McKay NP, Kaufman DS (2014) Sci Data 1, doi: 10.1038/ sdata.2014.26

PAGES 2k Consortium (2013) Nat Geo 6: 339-346

Tingley MP et al. (2012) Quat Sci Rev 35: 1-22 